\title{
Are You Ready to Take Your Drug Product from Phase I Studies to the Subsequent Phases?
}

\author{
Francois-Xavier Lacasse*1 and Stephane Lamouche ${ }^{2}$ \\ ${ }^{1}$ Faculty of Pharmacy, University of Montreal, 2940 Chemin de Polytechnique,Montreal, Quebec, Canada \\ ${ }^{2}$ SyneosHealthTM, 5160 Boulevard Decarie bureau 800, Montreal, Quebec, Canada
}

Received: June 16, 2018; Published: June 20, 2018

*Corresponding author: Francois-Xavier Lacasse, Faculty of Pharmacy, University of Montreal, 2940 Chemin de Polytechnique, Montreal, Quebec, Canada, Email: pharmfx@videotron.ca

\section{Short Communication}

Building a safety assessment package to go into the Phase I clinical stage sounds simple enough. All the steps pertaining to preclinical testing, pharmaceutical development, clinical supplies manufacturing, and regulatory submissions are all clearly defined within a repertoire of guidelines that outline the acceptable requirements. The timelines to enter into Phase I clinical trials are becoming increasingly shorter as companies seek definitive results that would prove or disprove their drug's potential. Gathering the maximum of information with regards to safety, efficacy and galenic aspects of the drug during early stages of drug development is often a challenge when faced with stresses of aggressive timelines, financial constraints and investor milestones. Since the cost associated with clinical trials accounts for a significant portion of the overall drug development costs, it is not surprising that the main focus is to ensure that the clinical trial is designed and developed to as near perfection as possible. Most of the time however, the other half of the project (i.e. the development of the actual drug product), is not given the same level of attention in spite of the increasing poor "druggability" properties of modern-day APIs. The quest of the nanomolar efficacy may be held responsible for that lack of optimal pharmaceutical properties. The downside with such an approach is that clinical testing may be initiated with a less than optimal formulation for which reliability, reproducibility, and scalability are not fully understood. Keeping in mind that the focus of Phase I testing is mainly to evaluate safety, additional bridging studies maybe needed later on when clinical results turns out to be promising. The

question that arises is: does this really accelerate the overall development process?

\section{Preclinical Phases: Drug Discovery-Medicinal Chemistry}

a. Thousands of molecules are discovered every year with the help of the combinatory chemistry and the high throughput screening. b. The API does not only represent the beginning of development but remains the most important ingredient of a formulation.

c. For this reason, it should be characterized as best as possible prior to being selected for "full development".

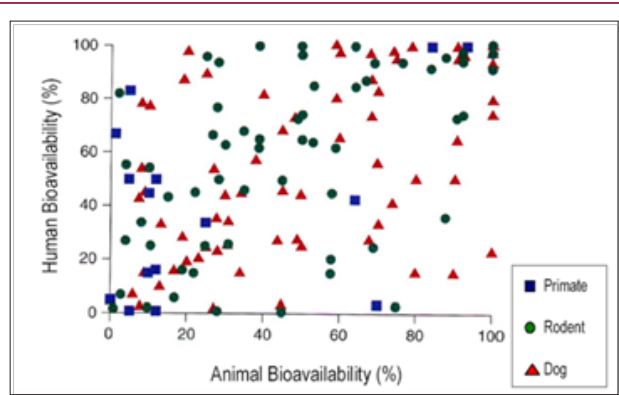

Figure 1: Correlation between animal and human bioavailability (Grass, G.M. and Sinko, P.J. Effect of Diverse Datasets on the Predictive Capability of ADME Models in Drug Discovery, DDT 6(12) (Suppl.) S54-S61 (2001)).

\section{In Vitro Bioavailability Prediction:}

a. To accelerate as much as possible the bioavailability prediction of an NME, a lot of IV/IVc equipments/tests are available but to date, such as dissolution apparatus type 1 to 4 and Caco- 2 cells to name just a few, it has already been proven that bioequivalence tests could not be achieved even though formulation demonstrated superimposed dissolution profiles (f2 higher than 50) and permeation pathways, between brand formulations and generic ones. The above remarks tend to show that in vitro equipments, even though have demonstrated powerful, very helpful, and cost saving results for high throughput screening were not able to discriminate the human in vivo behavior of two pharmaceutically equivalent drug products. 
b. In parallel, similar comment for the in vitro prediction of bioavailability above can be said for the in vivo predictability. The distribution of the dots in the Figure 1 shows that the best animal model for the human remains the human.

\section{Why is Preformulation so Important?}

Sir Arthur Conan Doyle (Sherlock Holmes) in the book "A Scandal in Bohemia" mentioned that"It is a capital mistake to theorize before one has data". Based on that assumption, it more than recommended to perform a thorough preformulation work since it is the foundation of developing robust formulations.

Preformulation phases: physical and chemical characterization of the API

a. Solubility,

b. Amorphous and crystalline states (polymorphism),

c. Solvated and hydrated states,

d. Hygroscopicity,

e. Particle size and particle size distribution,

f. Salts versus parent molecule,

g. Chirality

All these characteristics above are closely connected with the API's activity

\section{Preformulation Phases: Selection and Optimization of the API}

a. The objective is to demonstrate the reliability of both the chemical (yield, purity) and the physical characteristics (polymorphism) of the API irrespective of the batch size.

b. The success of the feasibility or proof-of-concept studies does not mean that the API is stable!

c. Be sure that the same polymorphic form/particle size distribution will be used for preclinical and clinical phases.

d. This should lead to a better reproducibility of the PK/PD of the drug substance from the bench to the Phase I scale.

e. Once a crystal shape is selected, a robust and reliable crystallization process should be developed and scalable.

f. Polymorphism could occur during formulation development, during stability studies, and even after marketing :

E.g. Ritonavir®: new form after 2 years, recall the original formulation from the market).

g. Key words: reliability \& reproducibility.

Other Properties of the API that are Dependent on its Solid-State:
a. Dissolution rate
b. Chemical stability
c. Melting point
d. Particle size/Shape
e. Hygroscopicity
f. Filterability
g. Suspension viscosity
h. Solubility rate
i. Bioavailability
j. Flowability
k. Compressibility
l. Bulk and Tap Density
m. Tablet hardness
n. Color

\section{The CMC Phases: Formulation Development}

a. Flexibility in the formulation and the manufacturing of Phase I clinical supplies because doses are still not defined in Phase I clinical trials.

b. This flexibility should not change the PK profile! Other CMC considerations to keep in mind:

a. Excipients are not necessarily inactive and should be selected carefully.

b. The dosage form and route of administration intended to be used in clinic should be determined ASAP and should be feasible and transferable from the pre-clinical/pilot steps to the clinical/pivotal studies.

c. Packaging is extremely important (neglected!).

\section{Absorption, Distribution, Metabolism, Excretion (ADME)}

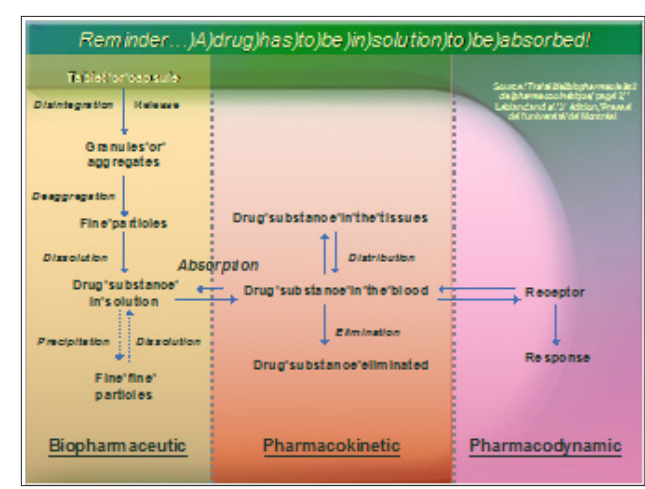

Figure 2: Schematic Biopharmaceutic, Pharmacokinetic and Pharmcodynamic phases.

Since the new molecular entities (small molecule) are getting more and more hydrophobic, the above CMC phase becomes tremendously important to get a stable, reliable and potent safety and efficacy profiles. The Figure 2 illustrates the three (3) phases a drug substance will have to go through to get these good profiles. In other words, if there is no A, there is no DME! Because of the 
poor solubility and permeation profiles, it can be understood that the biopharmaceutical phase, the phase where the drug substance should become bioavailable, has then become the cornerstone of good pharmacokinetical and pharmacodynamical behaviours.

\section{Conclusions and Final Thoughts}

a. Few chemical entities are readily druggable (POC are most of the time done with liquid solution/suspension).

b. Solid-state chemistry should ultimately drive the API selection:

Decision based on the best physico-chemical characteristics (the best candidate may often be the best compromise). c. Given its direct impact on the overall development program, it is imperative that a thorough early drug development approach with a long-term view be adopted and integrated as early as possible.

d. Each scientific discipline (pre-clinical, pharmaceutical $\mathrm{R} \& \mathrm{D}$, clinical, etc.) should work very closely in parallel, not sequentially, in order to maximize your chances of not only bringing your new drug successfully into clinic, but also to anticipate and plan for challenges that may come in later phases of development.

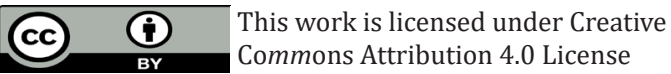

Submission Link: https://biomedres.us/submit-manuscript.php

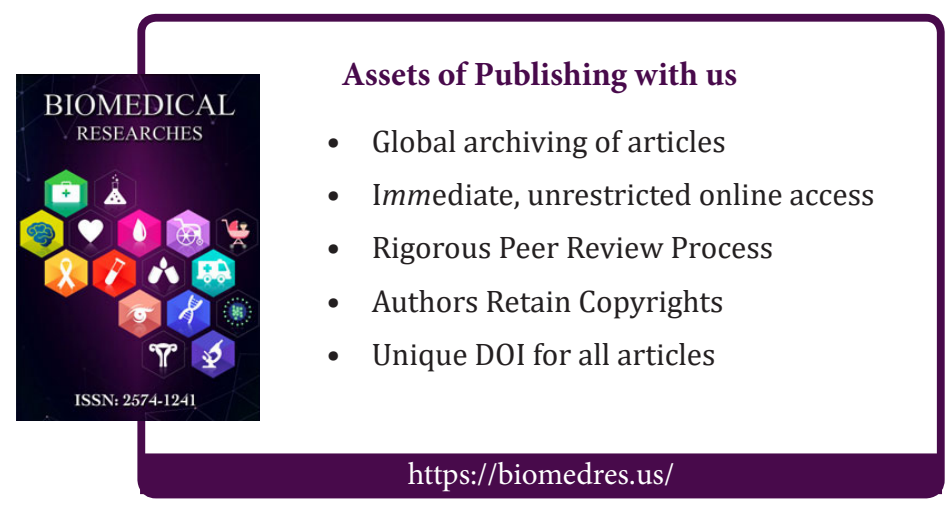

